\title{
Comparison of survival rates between patients treated with conventional radiotherapy and helical tomotherapy for head and neck cancer
}

\author{
Moonkyoo Kong, MD, Seong Eon Hong, MD, Jinhyun Choi, MD, Youngkyong Kim, MD \\ Department of Radiation Oncology, Kyung Hee University School of Medicine, Seoul, Korea
}

\begin{abstract}
Purpose: Compared to conventional radiotherapy (RT), intensity-modulated radiotherapy (IMRT) significantly reduces the rate of treatment-induced late toxicities in head and neck cancer. However, a clear survival benefit of IMRT over conventional RT has not yet been shown. This study is among the first comparative study to compare the survival rates between conventional RT and helical tomotherapy in head and neck cancer.

Materials and Methods: From January 2008 to November 2011, 37 patients received conventional RT and 30 patients received helical tomotherapy for management of head and neck cancer. We retrospectively compared the survival rates between patients treated with conventional RT and helical tomotherapy, and analyzed the prognostic factors for survival.

Results: The 1- and 2-year locoregional recurrence-free survival rates were $61.2 \%$ and $58.1 \%$ for the conventional RT group, $89.3 \%$ and $80.3 \%$ for the helical tomotherapy group, respectively. The locoregional recurrence-free survival rates of the helical tomotherapy group were significantly higher than conventional RT group $(p=0.029)$. There were no significant differences in the overall and distant metastasis-free survival between the two groups. RT technique, tumor stage, and RT duration were significant prognostic factors for locoregional recurrence-free survival.

Conclusion: This study showed the locoregional recurrence-free survival benefits of helical tomotherapy in the treatment of head and neck cancers.
\end{abstract}

Keywords: Helical tomotherapy, Conventional radiotherapy, Survival rate, Head and neck cancer

\section{Introduction}

Radiotherapy is the main non-surgical treatment for head and neck cancer. In recent years, intensity-modulated radiotherapy (IMRT) has become widely adopted for radiotherapeutic management of head and neck cancer because of its ability to achieve a highly conformal dose distribution. The computercontrolled linear accelerators and multileaf collimators used with this technique permit the administration of a highly conformal dose over that achieved with conventional radiotherapy (RT) [1]. Also, this allows better sparing of the organs at risk, leading to a decrease in acute and late side effects [2]. Theoretically, IMRT may improve primary tumor control and subsequent survival by allowing for either accelerated fractionation or fewer treatment interruptions due to a reduction in acute toxicity $[3,4]$. However, there is

Received 12 October 2012, Revised 27 November 2012, Accepted 14 December 2012.

Correspondence: Seong Eon Hong, MD, Department of Radiation Oncology, Kyung Hee University Medical Center, 23 Kyungheedae-gil, Dongdaemoon-gu, Seoul 130-702, Korea. Tel: +82-2-958-8661, Fax: +82-2-958-9469, E-mail: anjdixn@ naver.com

(c) This is an Open Access article distributed under the terms of the Creative Commons Attribution Non-Commercial License (http://creativecommons.org/ licenses/by-nc/3.0/) which permits unrestricted non-commercial use, distribution, and reproduction in any medium, provided the original work is properly cited.

www.e-roj.org 
theoretically also a higher risk of local relapse because the sharp dose gradient fall-off of an IMRT plan increases the likelihood of a geographical miss [5]. Therefore, assessing the clinical outcome of IMRT for head and neck cancer patients, in terms of local control and survival, is essential.

Several studies have shown consistently improved dosimetric parameters in IMRT than in conventional radiotherapy (RT) plans such as 2-dimensional conventional RT or 3-dimensional conformal RT, in head and neck cancer $[6,7]$. In addition, several published studies have reported that the use of IMRT for head and neck cancer significantly reduces the rate of treatment-induced toxicities [8-10], and improves quality of life $[8,11,12]$. However, a clear survival benefit of IMRT over conventional RT has not yet been shown, and findings regarding survival, tumor control, or other treatment efficacy remain generally inconclusive. In this study, we compared the survival rates of patients treated for head and neck cancer with either conventional RT or helical tomotherapy based IMRT, and analyzed the prognostic factors for survival in patients with head and neck cancer.

\section{Materials and Methods}

\section{Patient population}

Eligibility criteria were: 1) histologically confirmed head and neck cancer, 2) treatment with RT either definitively or postoperatively, 3) good general condition with performance status of $\leq 2$ in the Eastern Cooperative Oncology Group classification, 4) no previous head and neck irradiation, 5) no previous or concurrent illness that would compromise completion of treatment, 6) no distant metastases, and 7) a follow-up more than 6 months after RT. At our institution, helical tomotherapy was started from January 2008. From January 2008 to November 2011, 98 patients received RT for treatment of head and neck cancer. Of these patients, 67 patients met the eligibility criteria and were included in this study.

The pretreatment evaluation consisted of a complete history and physical examination, pan-endoscopy, complete blood counts, liver and renal function tests, chest $\mathrm{X}$-rays, dental evaluations, and computed tomography (CT) scans and magnetic resonance imaging (MRI) of the head and neck region. Positron emission tomography (PET), bone scans, and CT scans of the abdomen and/or chest were obtained when clinically indicated. All patients were staged in accordance with the American Joint Committee on Cancer (AJCC) TNM staging system (7th edition). Patients treated with definitive RT were staged clinically, while the pathological stage was applied to postoperative cases. The histologic grade was described according to the World Health Organization classification. Institutional Review Board approval was obtained for the review and analysis of patient data.

\section{Treatments}

All patients underwent CT-planned RT with either 3-dimensional conformal RT (conventional RT) or helical tomotherapy. The choice between conventional RT and helical tomotherapy was determined by the radiation oncologist, who also took the patient's interests into account. The gross tumor volume (GTV) was defined as the gross extent of the primary tumor and grossly involved lymph nodes, as demonstrated by imaging studies and physical examination. The high-risk clinical target volume (CTV1) was defined as the GTV plus a 1 to $1.5 \mathrm{~cm}$ margin to account for subclinical tumor spread. In postoperative situations, the CTV1 was defined as surgical bed plus a 0.5 to $1 \mathrm{~cm}$ margin to account for any microscopic residual disease. For both definitive and postoperative situations, the low-risk clinical target volume (CTV2) was defined as prophylactically treated cervical or supraclavicular lymph nodes. The planning target volume (PTV) was derived from an automated $0.5 \mathrm{~cm}$ three-dimensional expansion of the CTV to account for setup errors. The delineated organs at risk were the spinal cord, brain stem, parotid glands, and pharyngeal constrictor. Other organs at risk, such as the optic chiasm, optic nerve, or lens, were identified according the physician's discretion. The maximum dose constraints were designed to be less than $45 \mathrm{~Gy}$ to the spinal cord and less than 50 Gy to the brain stem. For parotid glands, the objective of dose constraints was to obtain a mean dose to the both parotid glands below 26 Gy whenever possible. For pharyngeal constrictor, the objective of dose constraints was to keep a mean dose below $50 \mathrm{~Gy}$. No overlap between the target volume and the organs at risk volume was permitted for optimization purposes. The plans were evaluated both quantitatively with dose-volume histograms and qualitatively by visually inspecting isodose curves. In general, treatment plans were considered acceptable if 1) the PTV was covered by $95 \%$ of isodose curves, 2) inhomogeneity of the PTV ranged from 95\% to $107 \%$, and 3 ) doses to normal organs were limited in their tolerances.

Conventional RT was delivered by a Clinac iX (Varian Medical System Inc., Palo Alto, CA, USA). All patients rested comfortably 
on a head support and were immobilized with thermoplastic masks from head to shoulder, and then were aligned with wall-mounted red lasers using external marks. In definitive situations, the PTV1 were treated with a total dose of 66-70 Gy and a daily dose of 2 Gy given on Monday through Friday. In postoperative situations, the PTV1 were treated with $60 \mathrm{~Gy}$ in 30 fractions unless there was residual disease, in which case 66-70 Gy with a daily 2 Gy was given. PTV2 were treated with $50 \mathrm{~Gy}$ in 25 daily fractions. Usually, patients were treated with bilateral opposing fields to the primary tumor and upper neck lymph nodes, and abutting anterior low neck field to cover the lower neck and supraclavicular lymph nodes. After the spinal cord had received the maximum tolerable dose, the bilateral opposing fields were coned down to the PTV1. If necessary, posterior neck lymph nodes were boosted with an electron beam.

Helical tomotherapy was delivered by TomoTherapy (TomoTherapy Inc., Madison, WI, USA). All patients were immobilized with head support, posterior vacuum bags and thermoplastic masks, and then were aligned with daily megavoltage CT images and wall-mounted red lasers. All patients were treated by a simultaneous integrated boost technique, and IMRT inverse planning was generated using the $\mathrm{Hi} \cdot$ Art Planning Station (TomoTherapy Inc.). To avoid low or high dose at fields junction area, split-field technique was not applied. The prescription dose was decided by the physician's judgment, and depended on the clinical or pathologic tumor staging, the patient's general condition, and the probability of RT-induced normal tissue toxicity. In definitive situations, daily doses of 1.8-2.25 Gy and a total dose of 66-73.5 Gy were delivered, and in postoperative situations, daily doses of 1.8-2.2 Gy and a total dose of 55-72.6 Gy were delivered to the PTV1 at 5 fractions per week. The PTV2 were treated with daily doses of 1.65-2.1 Gy and a total dose of 45-63 Gy. The biologically effective dose was calculated using a linear quadratic model with respect to acute effects on the tumor, with an $\alpha / \beta$ ratio of 10 [13].

The type of surgery used depended on the primary site, the tumor extent, cosmetic considerations, and the discretion of surgeon. In general, an attempt was made to maximize local control with preservation of functional and cosmetic outcomes. Reconstructive surgery was subsequently performed by plastic surgeons depending on the tissue defect in the operative region. Patients were referred for RT if highrisk pathologic features such as extracapsular extension, positive surgical margins, multiple lymph node involvement, or lymphovascular space invasion were found.

The implementation of chemotherapy depended on clinical or pathologic staging, the presence of high risk pathologic features, and the patient's performance status. The most common concurrent chemotherapy regimen was cisplatin (100 $\mathrm{mg} / \mathrm{m}^{2}$ ) for three cycles during RT. The regimen of chemotherapy was individualized based on the patient's general condition and compliance.

\section{Evaluation}

Patients were examined at least weekly during RT to monitor radiation-induced acute toxicity. After completion of RT, patients were evaluated every 2 to 4 weeks until the acute toxicities subsided. Routine physical examinations and blood work were done every 4 to 8 weeks for the first 6 months, and then every 3 to 6 months thereafter. CT scans or MRIs of the head and neck were obtained within 2 to 3 months after RT and then yearly or when clinically indicated.

All events were measured from the last day of radiation therapy. Locoregional recurrence was defined as an increase of the size of target lesions or the appearance of new lesions in the head and neck region as diagnosed by clinical or radiologic examination. Distant metastasis was defined as evidence of tumor in any other area. The locoregional recurrence were classified as 1) in-field, in which 95\% or more of the recurrence volume was within the $95 \%$ isodose, 2) marginal, in which 20\% to 95\% of the recurrence volume was within the 95\% isodose, and 3) out-field, in which less than 20\% of recurrence volume was within 95\% isodose [14]. Survival was determined from the last day of radiation therapy to the date of death or, in the case of survivors, to the date of the last follow-up.

Acute and late normal tissue toxicities were graded according to the Radiation Therapy Oncology Group (RTOG)/European Organization for the Treatment of Cancer (EORTC) radiation toxicity criteria. Acute toxicities were defined as toxicities that occurred during RT or within 3 months after completion of RT. Toxicities that occurred thereafter were graded as late toxicities.

\section{Statistical analysis}

The two groups (conventional RT group and helical tomotherapy group) were compared by chi-square test to detect differences in proportion. Survival rates were estimated by the Kaplan-Meier method and compared using a log-rank test. Parameters evaluated as potential prognostic factors of 
survival were: RT technique, age, gender, performance status, tumor cell type, tumor cell differentiation, AJCC stage, RT aim (definitive vs. postoperative), chemotherapy, RT dose, interruption of RT, and RT duration. All parameters were categorized into two groups according to patient distribution, and further assessed in a multivariate analysis, using a Cox regression hazard model. All analyses were performed using SPSS ver. 18.0 (SPSS Inc., Chicago, IL, USA). A p-value $<0.05$ was considered statistically significant.

\section{Results}

\section{Patient and disease characteristics}

The median age of all patients was 58.6 years (range, 20.1 to 84.5 years). There were 48 male (71.6\%) and 19 female patients (28.4\%). The performance status was 0 for 3 patients (4.5\%), 1 for 36 patients (53.7\%), 2 for 28 patients (41.8\%). Tumor sites were the oral cavity for 25 patients $(37.3 \%)$, the nasopharynx for 13 patients (19.4\%), the oropharynx for 9 patients (13.4\%), the larynx for 9 patients (13.4\%), the hypopharynx for 7 patients (10.4\%), and the paranasal sinus for 4 patients (6.0\%). According to 2010 AJCC classification, 4 patients (5.9\%) were stage I, 11 patients (16.4\%) were stage II, 16 patients (23.9\%) were stage III, 33 patients (89.2\%) were stage IVA, and 3 patients (4.5\%) were stage IVB. Twenty-eight patients (41.8\%) received definitive RT and 39 patients (58.2\%) received definitive surgery followed by postoperative RT. Concurrent chemotherapy was given to 24 patients (35.8\%), and 15 patients (22.4\%) had temporarily interrupted RT because of acute toxicities or refusal of treatment. The median follow-up duration of all patients was 23.5 months (range, 6.0 to 57.2 months).

Of all patients, 37 patients received conventional RT and 30 patients received helical tomotherapy. Patient and disease characteristics are summarized in Table 1. There were more patients who received definitive RT and higher RT dose in the helical tomotherapy group. However, there were no significant differences in other characteristics between the two groups.

\section{Treatment outcomes and prognostic factors}

The median follow-up time of the conventional RT group and the helical tomotherapy group was 23.1 and 24.1 months, respectively. During the follow-up period, 29 patients (78.4\%) from the conventional RT group and 28 patients (93.3\%) from the helical tomotherapy group were still alive. The 1 and 2 -year overall survival rates were $93.9 \%$ and $87.1 \%$ for the conventional RT group, $96.6 \%$ and $96.6 \%$ for the helical tomotherapy group, respectively. The overall survival rates of the helical tomotherapy group were higher than the conventional RT group, however, there were no significant differences in the overall survival between the two groups $(p=$ 0.095) (Fig. 1).

During the follow-up period, 18 patients (48.6\%) from the conventional RT group and 7 patients (23.3\%) from the helical tomotherapy group developed locoregional recurrence. Among the 18 patients who experienced locoregional recurrence after conventional RT, 10 patients developed in-field, 4 patients developed marginal, and 3 patients developed out-field locoregional recurrence. The remaining 1 patient developed both marginal and out-field locoregional recurrence. Among the 7 patients who experienced locoregional recurrence after helical tomotherapy, 2 patient developed in-field and 3 patients developed out-field locoregional recurrence. The remaining 2 patients developed both in-field and marginal locoregional recurrence. The 1- and 2-year locoregional recurrence-free survival rates were $61.2 \%$ and $58.1 \%$ for the conventional RT group, $89.3 \%$ and $80.3 \%$ for the helical tomotherapy group, respectively. The locoregional recurrence-free survival rates of the helical tomotherapy group were significantly higher than conventional RT group ( $p=0.029$ ) (Fig. 2).

During follow-up period, 7 patients (18.9\%) from the conventional RT group and 5 patients (16.7\%) from the helical tomotherapy group developed distant metastases. The most common metastatic site was the lung. Among the 7 patients who experienced distant metastases after conventional RT, 6 patients developed distant metastases in the lung, and 1 patient in the bone. Five patients experienced both locoregional and distant recurrence. Among the 5 patients who experienced distant metastases after helical tomotherapy, 2 patients developed distant metastases in the bone, 1 patient in the lung, 1 patient in the axilla, and 1 patient in the brain. Two patients experienced both locoregional and distant recurrence. The 1- and 2-year distant metastasis-free survival rates were $86.1 \%$ and $82.4 \%$ for the conventional RT group, $92.0 \%$ and $75.1 \%$ for the helical tomotherapy group, respectively ( $p=$ 0.994) (Fig. 3).

In all patient group, univariate and multivariate analyses were performed to identify prognostic factors for survival. In both univariate and multivariate analyses, there was no significant prognostic factor for overall survival (Table 2). For locoregional recurrence-free survival, RT technique, AJCC tumor stage, and RT duration were significant prognostic 
Table 1. Patient characteristics

\begin{tabular}{|c|c|c|c|}
\hline Characteristic & Conventional RT $(n=37)$ & Helical tomotherapy $(n=30)$ & $p$-value \\
\hline Age (yr) & $63.4(20.1-84.3)$ & $56.5(29.3-84.5)$ & 0.090 \\
\hline$\leq 60$ & $17(45.9)$ & $20(66.7)$ & \\
\hline$>60$ & $20(54.1)$ & $10(33.3)$ & \\
\hline \multicolumn{4}{|l|}{ Gender } \\
\hline Male & $26(70.3)$ & $22(73.3)$ & 0.782 \\
\hline Female & $11(29.7)$ & $8(26.7)$ & \\
\hline \multicolumn{4}{|l|}{ ECOG performance score } \\
\hline $0-1$ & $24(64.9)$ & $15(50.0)$ & 0.220 \\
\hline 2 & $13(35.1)$ & $15(50.0)$ & \\
\hline \multicolumn{4}{|l|}{ Site } \\
\hline Oral cavity & $15(40.5)$ & $10(33.3)$ & 0.074 \\
\hline Nasopharynx & $3(8.1)$ & $10(33.3)$ & \\
\hline Oropharynx & $5(13.5)$ & $4(13.3)$ & \\
\hline Hypopharynx & $6(16.2)$ & $1(3.3)$ & \\
\hline Larynx & $5(13.5)$ & $4(13.3)$ & \\
\hline Paranasal sinus & $3(8.1)$ & $1(3.3)$ & \\
\hline \multicolumn{4}{|l|}{ Tumor cell type } \\
\hline Squamous cell carcinoma & $33(89.2)$ & $22(73.3)$ & 0.092 \\
\hline Other histology & $4(10.8)$ & $8(26.7)$ & \\
\hline \multicolumn{4}{|l|}{ Tumor cell differentiation } \\
\hline Well & $11(29.7)$ & $6(20.0)$ & 0.363 \\
\hline Moderate or poor & $26(70.3)$ & $24(80.0)$ & \\
\hline \multicolumn{4}{|l|}{ Tumor stage } \\
\hline $\mathrm{T} 1$ & $3(8.2)$ & $6(20.0)$ & 0.071 \\
\hline $\mathrm{T} 2$ & $13(35.1)$ & $17(56.7)$ & \\
\hline T3 & $11(29.7)$ & $3(10.0)$ & \\
\hline $\mathrm{T} 4$ & $10(27.0)$ & $4(13.3)$ & \\
\hline \multicolumn{4}{|l|}{ Nodal stage } \\
\hline No & $15(40.5)$ & $9(30.0)$ & 0.091 \\
\hline N1 & $3(8.1)$ & $7(23.3)$ & \\
\hline N2 & $18(48.6)$ & $14(46.7)$ & \\
\hline N3 & $1(2.8)$ & $0(0)$ & \\
\hline \multicolumn{4}{|l|}{ AJCC stage } \\
\hline$\leq|l|$ & $13(35.1)$ & $18(60.0)$ & 0.052 \\
\hline$\geq \mathrm{IVA}$ & $24(64.9)$ & $12(40.0)$ & \\
\hline \multicolumn{4}{|l|}{ RT aim } \\
\hline Definitive & $11(29.7)$ & $17(56.7)$ & 0.026 \\
\hline Postoperative & $26(70.3)$ & $13(43.3)$ & \\
\hline \multicolumn{4}{|l|}{ Concurrent chemotherapy } \\
\hline Yes & $10(27.0)$ & $14(46.7)$ & 0.095 \\
\hline No & 27 (73.0) & 16 (53.3) & \\
\hline RT dose (PTV1, Gy $\left.{ }_{10}\right)$ & $72.0(72.0-84.0)$ & $80.5(67.1-88.9)$ & 0.001 \\
\hline$\leq 75$ & $24(64.9)$ & $4(13.3)$ & \\
\hline$>75$ & $13(35.1)$ & $26(86.7)$ & \\
\hline \multicolumn{4}{|l|}{ Interruption of RT } \\
\hline Yes & $8(21.6)$ & $7(23.3)$ & 0.867 \\
\hline No & 29 (78.4) & $23(76.7)$ & \\
\hline RT duration (wk) & $7.0(6.0-8.8)$ & $7.0(5.1-10.2)$ & 0.479 \\
\hline$\leq 7$ & $19(51.4)$ & $18(60.0)$ & \\
\hline$>7$ & $18(48.6)$ & $12(40.0)$ & \\
\hline
\end{tabular}

Values are presented as median (range) or number (\%).

ECOG, the Eastern Cooperative Oncology Group; AJCC, the American Joint Committee on Cancer; RT, radiotherapy; PTV, planning target volume.

${ }^{\text {a) }}$ Chi-square test. 


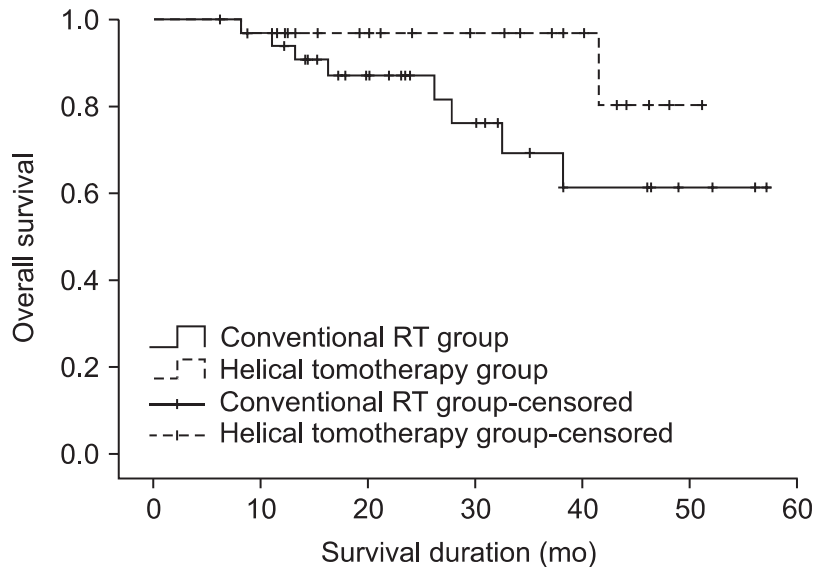

Fig. 1. Overall survival in the conventional radiotherapy (RT) group and the helical tomotherapy group. The 1- and 2-year overall survival rates were $93.9 \%$ and $87.1 \%$ for the conventional RT group, $96.6 \%$ and $96.6 \%$ for the helical tomotherapy group, respectively $(p=0.095)$.

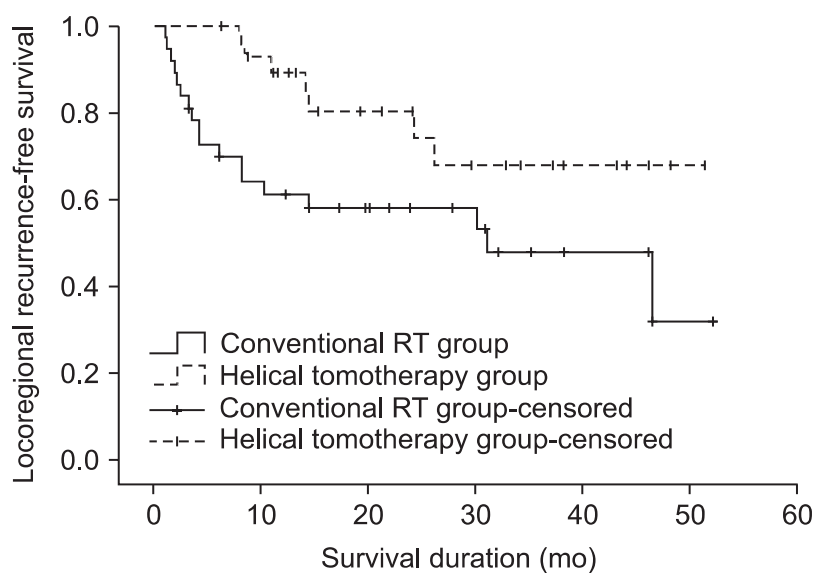

Fig. 2. Loco-regional recurrence-free survival in the conventional radiotherapy (RT) group and the helical tomotherapy group. The 1 - and 2-year loco-regional recurrence-free survival rates were $61.2 \%$ and $58.1 \%$ for the conventional RT group, 89.3\% and $80.3 \%$ for the helical tomotherapy group, respectively $(p=0.029)$.

factors in univariate analyses. In multivariate analyses, these 3 parameters remained significant factors for locoregional recurrence-free survival (Table 3). There was no significant prognostic factor for distant metastasis-free survival in univariate analyses. However, in multivariate analyses, the tumor cell type was significantly associated with distant metastasis-free survival (Table 4).

\section{Toxicities}

Because all patients were followed up for 6 months or more,

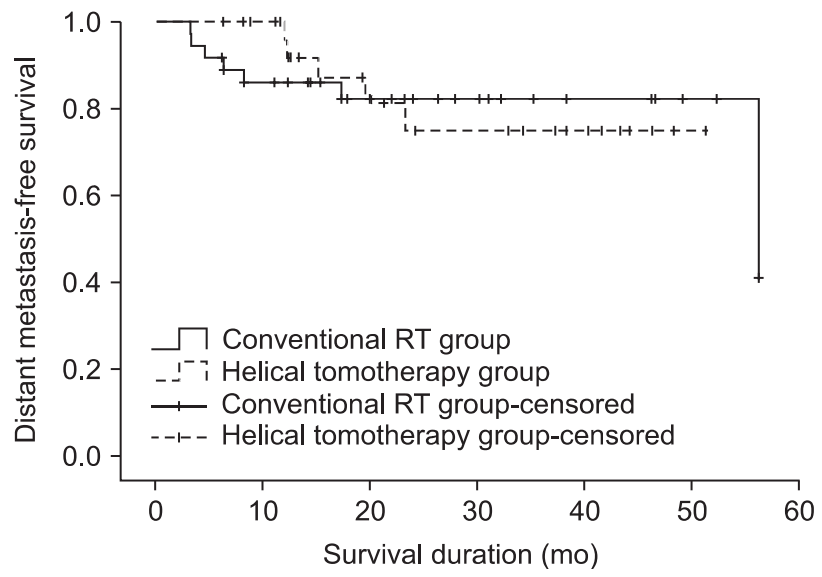

Fig. 3. Distant metastasis-free survival in the conventional radiotherapy (RT) group and the helical tomotherapy group. The 1 - and 2-year distant metastasis-free survival rates were $86.1 \%$ and $82.4 \%$ for the conventional RT group, $92.0 \%$ and $75.1 \%$ for the helical tomotherapy group, respectively ( $p=0.994)$.

radiation-induced toxicities could be evaluated in all patients. Acute toxicities occurred in almost all patients. The most common acute toxicities were mucositis, dysphagia, and xerostomia. There were no significant differences in acute toxicities between the conventional RT group and the helical tomotherapy group. The incidences of acute toxicities after RT are summarized in Table 5. The frequently encountered late toxicities were xerostomia and dysphagia. There were significantly higher incidences of Grade 2 or worse late xerostomia and dysphagia in patients receiving conventional $\mathrm{RT}$ ( $p=0.004$ for xerostomia and $p=0.020$ for dysphagia). The incidences of late toxicities after RT are summarized in Table 6 .

\section{Discussion and Conclusion}

Helical tomotherapy, a novel IMRT technique, utilizes a rotating gantry in which a 6-MV linear accelerator (Linac) is rotated continuously through $360^{\circ}$ around the patient, and a computer-controlled binary multileaf collimator [15]. Some studies have reported that helical tomotherapy based IMRT offers improved dose distribution and the potential to further improve the therapeutic ratio in the management of head and neck cancer [16-18]. However, few studies have reported the clinical outcomes of helical tomotherapy in terms of survival and tumor control [19]. Furthermore, to our knowledge, no studies have investigated the survival differences between conventional RT and helical tomotherapy based IMRT in head and neck cancer patients. This study is among the first 
Table 2. Analyses of prognostic factors for overall survival

\begin{tabular}{|c|c|c|c|c|c|}
\hline \multirow{2}{*}{ Variable } & \multirow{2}{*}{$\begin{array}{l}2-y r \text { overall } \\
\text { survival }(\%)\end{array}$} & \multirow{2}{*}{$\begin{array}{c}\text { Univariate } \\
\text { analysis } \\
\text { p-value }\end{array}$} & \multicolumn{3}{|c|}{ Multivariate analysis } \\
\hline & & & $H R$ & $95 \% \mathrm{Cl}$ & p-value \\
\hline $\begin{array}{l}\text { RT technique (conventional RT : helical } \\
\text { tomotherapy) }\end{array}$ & $87.1: 96.6$ & 0.095 & 0.590 & $0.740-4.732$ & 0.620 \\
\hline Age $(\leq 60$ yr $:>60 \mathrm{yr})$ & $88.5: 96.0$ & 0.732 & 1.178 & $0.315-4.405$ & 0.935 \\
\hline Gender (M : F) & $93.1: 85.2$ & 0.687 & 0.759 & $0.157-3.670$ & 0.865 \\
\hline ECOG performance score $(0-1: 2)$ & $97.1: 82.1$ & 0.553 & 1.444 & $0.412-5.509$ & 0.426 \\
\hline Tumor cell type (SqCC : other histology) & $93.6: 82.5$ & 0.977 & 0.994 & $0.202-4.880$ & 0.984 \\
\hline $\begin{array}{l}\text { Tumor cell differentiation (well : mod- } \\
\text { erate or poor) }\end{array}$ & $92.9: 90.7$ & 0.229 & 0.516 & $0.109-2.444$ & 0.404 \\
\hline AJCC stage $(\leq I I I: \geq I V A)$ & $93.0: 89.6$ & 0.320 & 1.681 & $0.365-7.735$ & 0.505 \\
\hline RT aim (definitive : postoperative) & $86.6: 94.3$ & 0.804 & 1.664 & $0.317-8.731$ & 0.654 \\
\hline Concurrent chemotherapy (no : yes) & $90.8: 91.7$ & 0.170 & 0.606 & $0.033-11.230$ & 0.737 \\
\hline RT dose (PTV1, Gy $\left.{ }_{10}\right)(\leq 75:>75)$ & $88.7: 92.7$ & 0.099 & 0.680 & $0.069-6.690$ & 0.741 \\
\hline Interruption of RT (no : yes) & $92.5: 85.7$ & 0.232 & 1.246 & $0.277-5.614$ & 0.774 \\
\hline RT duration ( $\leq 7 w k:>7 w k)$ & $93.3: 88.2$ & 0.104 & 4.016 & $0.807-19.991$ & 0.090 \\
\hline
\end{tabular}

$\mathrm{HR}$, hazard ratio; $\mathrm{Cl}$, confidence interval; RT, radiotherapy; ECOG, Eastern Cooperative Oncology Group; SqCC, squamous cell carcinoma; AJCC, American Joint Committee on Cancer; PTV, planning target volume.

Table 3. Analyses of prognostic factors for loco-regional recurrence-free survival

\begin{tabular}{|c|c|c|c|c|c|}
\hline \multirow{2}{*}{ Variable } & \multirow{2}{*}{$\begin{array}{c}\text { 2-yr locoregional } \\
\text { recurrence-free } \\
\text { survival }(\%)\end{array}$} & \multirow{2}{*}{$\begin{array}{c}\text { Univariate } \\
\text { analysis } \\
\text { p-value }\end{array}$} & \multicolumn{3}{|c|}{ Multivariate analysis } \\
\hline & & & $H R$ & $95 \% \mathrm{Cl}$ & p-value \\
\hline $\begin{array}{l}\text { RT technique (conventional RT : helical } \\
\text { tomotherapy) }\end{array}$ & $58.1: 80.3$ & 0.029 & 0.203 & $0.079-1.841$ & 0.048 \\
\hline Age $(\leq 60 \mathrm{yr}:>60 \mathrm{yr})$ & $69.4: 66.1$ & 0.394 & 1.457 & $0.335-2.162$ & 0.628 \\
\hline Gender (M : F) & $68.6: 67.7$ & 0.961 & 1.337 & $0.442-4.291$ & 0.954 \\
\hline ECOG performance score $(0-1: 2)$ & $69.9: 65.9$ & 0.520 & 0.913 & $0.325-2.561$ & 0.427 \\
\hline Tumor cell type (SqCC : other histology) & $65.0: 82.5$ & 0.347 & 1.728 & $0.403-7.411$ & 0.730 \\
\hline $\begin{array}{l}\text { Tumor cell differentiation (well : moder- } \\
\text { ate or poor) }\end{array}$ & $61.2: 70.2$ & 0.961 & 0.575 & $0.178-1.854$ & 0.721 \\
\hline AJCC stage $(\leq \mathrm{III}: \geq \mathrm{IVA})$ & $79.1: 59.5$ & 0.012 & 3.074 & $1.226-7.706$ & 0.005 \\
\hline RT aim (definitive : postoperative) & $60.7: 73.1$ & 0.245 & 0.150 & $0.024-0.937$ & 0.139 \\
\hline Concurrent chemotherapy (no : yes) & $65.1: 73.8$ & 0.441 & 0.293 & $0.078-1.103$ & 0.360 \\
\hline RT dose (PTV1, Gy $\left.{ }_{10}\right)(\leq 75:>75)$ & $63.0: 71.6$ & 0.541 & 0.432 & $0.092-2.036$ & 0.364 \\
\hline Interruption of RT (no : yes) & $71.1: 58.7$ & 0.773 & 0.920 & $0.300-2.819$ & 0.517 \\
\hline RT duration ( $\leq 7 w k:>7 w k)$ & $79.1: 54.8$ & 0.013 & 2.995 & $1.316-6.813$ & 0.007 \\
\hline
\end{tabular}

$\mathrm{HR}$, hazard ratio; $\mathrm{Cl}$, confidence interval; RT, radiotherapy; ECOG, the Eastern Cooperative Oncology Group; SqCC, squamous cell carcinoma; AJCC, the American Joint Committee on Cancer; PTV, planning target volume.

comparative study to compare the survival rates between patients treated with either conventional RT or helical tomotherapy for head and neck cancer.

Several studies compared the survival rates between conventional RT and Linac-based IMRT for head and neck cancer $[10,20-26]$. The majority of studies showed no significant differences in survival rates between the two RT techniques [10,20,22-25]. The parotid-sparing intensity modulated versus conventional radiotherapy in head and neck cancer (PARSPORT) randomized trial, the first multi-institutional prospective trial comparing IMRT with conventional RT for head and neck cancer, compared the 
Table 4. Analyses of prognostic factors for distant metastasis-free survival

\begin{tabular}{|c|c|c|c|c|c|}
\hline \multirow{2}{*}{ Variable } & \multirow{2}{*}{$\begin{array}{l}\text { 2-yr distant metasta- } \\
\text { sis-free survival (\%) }\end{array}$} & \multirow{2}{*}{$\begin{array}{c}\text { Univariate } \\
\text { analysis } \\
\text { p-value }\end{array}$} & \multicolumn{3}{|c|}{ Multivariate analysis } \\
\hline & & & $H R$ & $95 \% \mathrm{Cl}$ & p-value \\
\hline $\begin{array}{l}\text { RT technique (conventional RT : } \\
\text { helical tomotherapy) }\end{array}$ & $82.4: 75.1$ & 0.994 & 1.164 & $0.308-4.396$ & 0.824 \\
\hline Age $(\leq 60 \mathrm{yr}:>60 \mathrm{yr})$ & $74.6: 87.1$ & 0.356 & 0.650 & $0.163-2.595$ & 0.542 \\
\hline Gender (M : F) & $79.1: 81.2$ & 0.888 & 0.968 & $0.232-4.039$ & 0.624 \\
\hline ECOG performance score (0-1:2) & $77.8: 81.8$ & 0.939 & 0.926 & $0.220-3.092$ & 0.257 \\
\hline $\begin{array}{l}\text { Tumor cell type (SqCC : other } \\
\text { histology) }\end{array}$ & $84.0: 61.1$ & 0.124 & 7.260 & $1.237-42.630$ & 0.028 \\
\hline $\begin{array}{l}\text { Tumor cell differentiation (well : } \\
\text { moderate or poor) }\end{array}$ & $75.3: 80.5$ & 0.919 & 1.012 & $0.226-4.538$ & 0.824 \\
\hline AJCC stage $(\leq \mathrm{III}: \geq \mathrm{IVA})$ & $81.5: 75.7$ & 0.960 & 1.107 & $0.307-3.992$ & 0.549 \\
\hline RT aim (definitive : postoperative) & $78.9: 79.4$ & 0.793 & 0.862 & $0.107-6.948$ & 0.547 \\
\hline Concurrent chemotherapy (no : yes) & $76.3: 83.5$ & 0.432 & 0.192 & $0.026-1.399$ & 0.103 \\
\hline RT dose (PTV1, Gy $\left.{ }_{10}\right)(\leq 75:>75)$ & $77.7: 79.8$ & 0.468 & 0.496 & $0.113-2.179$ & 0.353 \\
\hline Interruption of RT (no : yes) & $79.0: 80.8$ & 0.661 & 0.603 & $0.114-3.186$ & 0.363 \\
\hline RT duration ( $\leq 7 w k:>7 w k)$ & $81.7: 75.9$ & 0.901 & 1.375 & $0.302-6.266$ & 0.569 \\
\hline
\end{tabular}

$\mathrm{HR}$, hazard ratio; $\mathrm{Cl}$, confidence interval; RT, radiotherapy; ECOG, Eastern Cooperative Oncology Group; SqCC, squamous cell carcinoma; AJCC, American Joint Committee on Cancer; PTV, planning target volume.

Table 5. Acute toxicities following radiotherapy for head and neck cancer

\begin{tabular}{|c|c|c|c|c|c|c|c|c|}
\hline \multirow{2}{*}{ Grade } & \multicolumn{4}{|c|}{ Conventional radiotherapy $(n=37)$} & \multicolumn{4}{|c|}{ Helical tomotherapy $(n=30)$} \\
\hline & 0 & 1 & 2 & 3 & 0 & 1 & 2 & 3 \\
\hline Mucositis & 0 & $6(16)$ & $12(32)$ & $19(51)$ & 0 & $4(13)$ & $11(37)$ & $15(50)$ \\
\hline Dysphagia & 0 & $6(16)$ & $15(41)$ & $16(43)$ & $1(3)$ & $5(17)$ & $12(40)$ & $12(40)$ \\
\hline Xerostomia & 0 & $20(54)$ & $12(32)$ & $5(14)$ & $1(3)$ & $9(30)$ & $15(50)$ & $5(17)$ \\
\hline
\end{tabular}

Values are presented as number (\%).

Table 6. Late toxicities following radiotherapy for head and neck cancer

\begin{tabular}{|c|c|c|c|c|c|c|c|c|}
\hline \multirow{2}{*}{ Grade } & \multicolumn{4}{|c|}{ Conventional radiotherapy $(n=37)$} & \multicolumn{4}{|c|}{ Helical tomotherapy $(n=30)$} \\
\hline & 0 & 1 & 2 & 3 & 0 & 1 & 2 & 3 \\
\hline Dysphagia & $13(35)$ & $4(11)$ & $16(43)$ & $4(11)$ & $13(43)$ & $10(33)$ & $4(13)$ & $3(10)$ \\
\hline Xerostomia & 0 & $2(5)$ & $26(70)$ & $9(24)$ & 0 & $18(60)$ & $9(30)$ & $3(10)$ \\
\hline
\end{tabular}

Values are presented as number (\%).

treatment outcomes of 94 patients treated by conventional RT (47 patients) and IMRT (47 patients) [10]. In that study, there were no differences in 2-year locoregional progression-free survival and overall survival. On the other hand, few studies reported significant differences in survival rates between the two RT techniques. Clavel et al. [21] compared the treatment outcomes of 249 oropharyngeal cancer patients treated by conventional RT (149 patients) and IMRT (100 patients), and reported significantly improved 3 -year overall survival ( $p<$ $0.001)$ and disease-free survival $(p=0.001)$ in patients treated by IMRT. In addition, Chao et al. [26] reported significantly improved 2-year disease-free $(p=0.002)$ and overall survival ( $p$ $=0.001)$ in oropharyngeal cancer patients treated by IMRT (12 patients) compared with patients treated by conventional RT (153 patients). In our study, we compared overall, locoregional recurrence-free, and distant metastasis-free survival between 
patients treated with conventional RT and helical tomotherapy for head and neck cancer. We could not find significant differences in overall or distant metastasis-free survival between the two groups. Although the overall survival rates of the helical tomotherapy group were relatively higher than the conventional RT group (2-year overall survival rates were $87.1 \%$ for the conventional RT group and $96.6 \%$ for the helical tomotherapy group), there was no significant difference between the two groups ( $p=0.095)$.

However, the locoregional recurrence-free survival rate in the helical tomotherapy group was significantly higher than in the conventional RT group (2-year locoregional recurrencefree survival rates were $58.1 \%$ for the conventional RT group and $80.3 \%$ for the helical tomotherapy group, $p=$ 0.029). In multivariate analyses of prognostic factors, helical tomotherapy was still associated with higher locoregional recurrence-free survival. Also, AJCC stage and RT duration were significant prognostic factors for locoregional recurrence-free survival in univariate and multivariate analyses.

Several studies have reported the survival rates in head and neck cancer patients after IMRT, and the reported 2- or 3 -year overall survival rates range from $67 \%$ to $92.1 \%$, the locoregional progression-free survival rates range from 64\% to $87 \%$, and the distant metastasis-free survival rates range from $76.3 \%$ to $92.7 \%$, respectively $[10,17,20-23,27-29]$. The Oncology and Radiotherapy Group for Head and Neck Cancer (GORTEC) 2004-03 prospective study reported the treatment outcomes of 208 head and neck cancer patients treated with IMRT in 8 centers [27]. Ninety-three patients (46\%) had postoperative IMRT and 78 patients (37.5\%) received concurrent chemotherapy. The estimated 2-year locoregional progression-free, metastatic progression-free, and overall survival rates were $86 \%, 92.7 \%$, and $86.7 \%$, respectively. The PARSPORT randomized trial also reported the survival rates of 47 oropharynx or hypopharynx cancer patients treated with IMRT in 6 centers, and the estimated 2-year locoregional progression-free and overall survival rates were both 78\% [10]. Chen et al. [19] reported a single-institutional study of helical tomotherapy for head and neck cancer. Of a total of 77 patients, 42 patients (55\%) received definitive helical tomotherapy, and 48 patients (62\%) received concurrent chemotherapy. The estimated 2-year disease-free, and overall survival rates were $71 \%$ and $82 \%$, respectively. In our study, 30 head and neck cancer patients were treated by helical tomotherapy, and the 2-year locoregional recurrence-free survival rates was $80.3 \%$, distant metastasis-free survival rates was 75.1\%, and overall survival rates was 96.6\%, respectively. Possible reasons for these inconsistent survival rates may stem from the wide variability in disease characteristics of the patients, differences in treatment strategy (such as definitive or postoperative RT, and RT alone or concurrent chemoradiotherapy), physicians' variability in the contouring of tumors and normal structures, and variable operators' experience in IMRT planning.

Usually, IMRT plans have steep dose gradients to deliver a highly conformal dose to target lesions and to spare the organs at risk. However, the sharp dose gradients may increase the likelihood of a geographical miss and the possibility of subsequent locoregional recurrence [5]. Therefore, it is important to examine the pattern of locoregional recurrence. In a retrospective study, Dawson et al. [14] analyzed failure patterns after IMRT in 58 head and neck cancer patients. Among 12 patients who suffered from locoregional recurrence, 10 patients (80\%) had in-field recurrence and the other 2 patients suffered from marginal recurrence. Chen et al. [19] also retrospectively analyzed failure patterns after helical tomotherapy in 77 head and neck cancer patients. Locoregional recurrence developed in 18 patients, and among these 18 patients, 12 patients (66.7\%) had in-field recurrence and 4 patients (22.2\%) had marginal recurrence. In our study, among 7 patients who suffered from locoregional recurrence after helical tomotherapy, 2 patients (28.6\%) had in-field recurrence and 2 patients (28.6\%) had both in-field and marginal recurrence. On the other hand, in the conventional RT group, among the 18 patients who developed locoregional recurrence, 15 patients (83.3\%) had in-field or marginal recurrence (in-field in 10 patients and marginal in 5 patients). Compared with other studies, in our study, in-field or marginal locoregional recurrence developed less frequently in the helical tomotherapy group, and helical tomotherapy did not increase the possibility of in-field or marginal locoregional recurrence when compared to conventional RT.

Several studies reported variable rates of late toxicities after IMRT in head and neck cancer patients, and the reported rates of grade 2 or worse late xerostomia and dysphagia range from $16 \%$ to $38 \%$, and $3 \%$ to $21 \%$, respectively $[10,19,24,26,27,30]$. However, regardless of the late toxicity rates, almost all studies reported that late toxicities were significantly less common in patients treated by IMRT than in the patients treated with conventional RT $[9,10,24,26]$. In our study, like other studies, there were significantly lower incidences of grade 2 or worse late xerostomia and dysphagia in patients receiving helical 
tomotherapy than in patients receiving conventional RT. The estimated rates of grade 2 or worse late xerostomia were $37 \%$ in the helical tomotherapy group and $94 \%$ in the conventional RT group, and the rates of grade 2 or worse late dysphagia were $23 \%$ in the helical tomotherapy group and $54 \%$ in the conventional RT group.

There were some limitations in this study. First, this study was retrospective, and therefore, may had some inherent bias. For example, the prescription dose of helical tomotherapy was decided according to physician's discretion rather than beforehand definite protocol, and the allocation of patients to either conventional or helical tomotherapy technique was not random. Second, the sample size was small, so we may not have detected small differences in survival. Third, the population studied was heterogenous. Fourth, evaluation of toxicities with RTOG/EORTC radiation toxicity criteria may be subjective and possibly under-or over-estimates the toxicities. And fifth, the duration of the follow-up period was not long, and consequently, this study may underestimate the recurrence rate. These limitations may make difficult to interpret the results obtained. However, as the first study which showed locoregional recurrence-free survival benefit of helical tomotherapy in head and neck cancer, we believe that this study contributes to resolving some inconclusive issues on radiotherapy in head and neck cancer management. If, in the near future, randomized prospective trial will be conducted with homogenous patient group, we will be able to draw more definite conclusion.

In conclusion, this study showed the locoregional recurrencefree survival benefits of helical tomotherapy in the treatment of head and neck cancers. In addition, we found that helical tomotherapy significantly decreased the occurrence of late xerostomia and dysphagia.

\section{Conflict of Interest}

No potential conflict of interest relevant to this article was reported.

\section{References}

1. Clark $\mathrm{CH}$, Bidmead $\mathrm{AM}$, Mubata $C D$, Harrington KJ, Nutting CM. Intensity-modulated radiotherapy improves target coverage, spinal cord sparing and allows dose escalation in patients with locally advanced cancer of the larynx. Radiother Oncol 2004;70:189-98.
2. Wu Q, Manning M, Schmidt-Ullrich R, Mohan R. The potential for sparing of parotids and escalation of biologically effective dose with intensity-modulated radiation treatments of head and neck cancers: a treatment design study. Int J Radiat Oncol Biol Phys 2000;46:195-205.

3. Orlandi E, Palazzi M, Pignoli E, Fallai C, Giostra A, Olmi P. Radiobiological basis and clinical results of the simultaneous integrated boost (SIB) in intensity modulated radiotherapy (IMRT) for head and neck cancer: a review. Crit Rev Oncol Hematol 2010;73:111-25.

4. Traynor AM, Richards GM, Hartig GK, et al. Comprehensive IMRT plus weekly cisplatin for advanced head and neck cancer: the University of Wisconsin experience. Head Neck 2010;32:599-606.

5. Bhide SA, Ahmed M, Newbold K, Harrington KJ, Nutting CM. The role of intensity modulated radiotherapy in advanced oral cavity carcinoma. J Cancer Res Ther 2012;8 Suppl 1:S67-71.

6. Chao KS, Low DA, Perez CA, Purdy JA. Intensity-modulated radiation therapy in head and neck cancers: the Mallinckrodt experience. Int J Cancer 2000;90:92-103.

7. Chau RM, Teo PM, Kam MK, Leung SF, Cheung KY, Chan AT. Dosimetric comparison between 2-dimensional radiation therapy and intensity modulated radiation therapy in treatment of advanced T-stage nasopharyngeal carcinoma: to treat less or more in the planning organ-at-risk volume of the brainstem and spinal cord. Med Dosim 2007;32:263-70.

8. Chao KS, Deasy JO, Markman J, et al. A prospective study of salivary function sparing in patients with head-and-neck cancers receiving intensity-modulated or three-dimensional radiation therapy: initial results. Int J Radiat Oncol Biol Phys 2001:49:907-16.

9. Rades D, Fehlauer F, Wroblesky J, Albers D, Schild SE, Schmidt R. Prognostic factors in head-and-neck cancer patients treated with surgery followed by intensity-modulated radiotherapy (IMRT), 3D-conformal radiotherapy, or conventional radiotherapy. Oral Oncol 2007:43:535-43.

10. Nutting $C M$, Morden JP, Harrington KJ, et al. Parotid-sparing intensity modulated versus conventional radiotherapy in head and neck cancer (PARSPORT): a phase 3 multicentre randomised controlled trial. Lancet Oncol 2011;12:127-36.

11. Vergeer MR, Doornaert PA, Rietveld DH, Leemans CR, Slotman BJ, Langendijk JA. Intensity-modulated radiotherapy reduces radiation-induced morbidity and improves health-related quality of life: results of a nonrandomized prospective study using a standardized follow-up program. Int J Radiat Oncol Biol Phys 2009;74:1-8.

12. Yao M, Karnell LH, Funk GF, Lu H, Dornfeld K, Buatti JM. Health-related quality-of-life outcomes following IMRT versus conventional radiotherapy for oropharyngeal squamous cell 
carcinoma. Int J Radiat Oncol Biol Phys 2007;69:1354-60.

13. Fowler JF. The linear-quadratic formula and progress in fractionated radiotherapy. Br J Radiol 1989;62:679-94.

14. Dawson LA, Anzai Y, Marsh L, et al. Patterns of localregional recurrence following parotid-sparing conformal and segmental intensity-modulated radiotherapy for head and neck cancer. Int J Radiat Oncol Biol Phys 2000;46:1117-26.

15. Mackie TR, Holmes T, Swerdloff $S$, et al. Tomotherapy: a new concept for the delivery of dynamic conformal radiotherapy. Med Phys 1993;20:1709-19.

16. Fiorino C, Dell'Oca I, Pierelli A, et al. Significant improvement in normal tissue sparing and target coverage for head and neck cancer by means of helical tomotherapy. Radiother Oncol 2006;78:276-82.

17. Yao M, Dornfeld KJ, Buatti JM, et al. Intensity-modulated radiation treatment for head-and-neck squamous cell carcinoma: the University of lowa experience. Int J Radiat Oncol Biol Phys 2005;63:410-21.

18. Sheng K, Molloy JA, Read PW. Intensity-modulated radiation therapy (IMRT) dosimetry of the head and neck: a comparison of treatment plans using linear accelerator-based IMRT and helical tomotherapy. Int J Radiat Oncol Biol Phys 2006;65:91723.

19. Chen $A M$, Jennelle $R L$, Sreeraman $R$, et al. Initial clinical experience with helical tomotherapy for head and neck cancer. Head Neck 2009;31:1571-8.

20. Chen AM, Li BO, Farwell DG, Marsano J, Vijayakumar S, Purdy JA. Improved dosimetric and clinical outcomes with intensitymodulated radiotherapy for head-and-neck cancer of unknown primary origin. Int J Radiat Oncol Biol Phys 2011;79: 756-62.

21. Clavel $S$, Nguyen $D H$, Fortin $B$, et al. Simultaneous integrated boost using intensity-modulated radiotherapy compared with conventional radiotherapy in patients treated with concurrent carboplatin and 5-fluorouracil for locally advanced oropharyngeal carcinoma. Int J Radiat Oncol Biol Phys 2012; 82:582-9.

22. Madani I, Vakaet L, Bonte K, Boterberg T, De Neve W. Intensity- modulated radiotherapy for cervical lymph node metastases from unknown primary cancer. Int J Radiat Oncol Biol Phys 2008;71:1158-66.

23. Fang FM, Chien $C Y$, Tsai WL, et al. Quality of life and survival outcome for patients with nasopharyngeal carcinoma receiving three-dimensional conformal radiotherapy vs. intensity-modulated radiotherapy-a longitudinal study. Int J Radiat Oncol Biol Phys 2008;72:356-64.

24. Chen WC, Hwang TZ, Wang WH, et al. Comparison between conventional and intensity-modulated post-operative radiotherapy for stage III and IV oral cavity cancer in terms of treatment results and toxicity. Oral Oncol 2009;45:505-10.

25. Yu JB, Soulos PR, Sharma R, et al. Patterns of care and outcomes associated with intensity-modulated radiation therapy versus conventional radiation therapy for older patients with head-and-neck cancer. Int J Radiat Oncol Biol Phys 2012;83:e101-7.

26. Chao KS, Majhail N, Huang CJ, et al. Intensity-modulated radiation therapy reduces late salivary toxicity without compromising tumor control in patients with oropharyngeal carcinoma: a comparison with conventional techniques. Radiother Oncol 2001;61:275-80.

27. Toledano I, Graff P, Serre A, et al. Intensity-modulated radiotherapy in head and neck cancer: results of the prospective study GORTEC 2004-03. Radiother Oncol 2012;103:57-62.

28. Lee N, Xia P, Fischbein NJ, Akazawa P, Akazawa C, Quivey JM. Intensity-modulated radiation therapy for head-andneck cancer: the UCSF experience focusing on target volume delineation. Int J Radiat Oncol Biol Phys 2003;57:49-60.

29. Chao KS, Ozyigit G, Tran BN, Cengiz M, Dempsey JF, Low DA. Patterns of failure in patients receiving definitive and postoperative IMRT for head-and-neck cancer. Int J Radiat Oncol Biol Phys 2003;55:312-21.

30. Van Gestel D, Van Den Weyngaert D, Schrijvers D, Weyler J, Vermorken JB. Intensity-modulated radiotherapy in patients with head and neck cancer: a European single-centre experience. Br J Radiol 2011;84:367-74. 